\title{
Surgical outcomes and prognostic factors for patients with gastric cancer spinal metastasis
}

This article was published in the following Dove Press journal:

Cancer Management and Research

\section{Nanzhe Zhong* \\ Ao Leng* \\ Shaohui He* \\ Minglei Yang \\ Dan Zhang \\ Jian Jiao \\ Wei Xu \\ Xinghai Yang \\ Jianru Xiao}

Department of Orthopedic Oncology and Spinal Tumor Center, Shanghai

Changzheng Hospital, Naval Medical

University, Shanghai 200003, People's

Republic of China

*These authors contributed equally to this work
Correspondence: Xinghai Yang; Jianru Xiao

Department of Orthopedic Oncology and Spinal Tumor Center, Changzheng Hospital, Naval Medical University, 4I5

Fengyang Road, Shanghai 200003, People's Republic of China

Tel +86 I 86I 618 1683:

$+86 \mid 3701785283$

Fax +8602181885634

Email cnspineyang@163.com;

jianruxiao83@163.com

\begin{abstract}
Purpose: To investigate the outcome and prognostic factors of surgery in treating gastric cancer spinal metastasis (GCSM).

Patients and methods: A total of 17 patients with GCSM who have undertaken spinal surgeries have been identified. Kaplan-Meier method and univariate analysis are adopted to investigate the prognostic factors affecting overall survival (OS) and progression-free survival (PFS).

Results: The median PFS and OS are 11.3 months (95\% CI: 7.8-14.9 months) and 11.9 months (95\% CI: 8.4-15.4 months), respectively. Postoperatively, all patients had substantial pain relief, with mean visual analog scale score descending from $6.6 \pm 1.6$ to $3.4 \pm 1.2$. Meanwhile, patients also showed improved neurological functions, with 8 of them having improvements of at least one level in Frankel classification. Univariate analysis presented that patients with carcinoembryonic antigen $(\mathrm{CEA})<6 \mu \mathrm{g} / \mathrm{L}(p=0.020)$, lactate dehydrogenase $(\mathrm{LDH})<300 \mathrm{U} / \mathrm{L}(p=0.012)$, alkaline phosphatase $(\mathrm{ALP})<200 \mathrm{U} / \mathrm{L}(p=0.007)$, and Tokuhashi score $>6(p=0.027)$ show longer OS. Moreover, application of bone cement, low level of ALP $(<200 \mathrm{U} / \mathrm{L})$, and LDH $(<300 \mathrm{U} / \mathrm{L})$ are associated with longer PFS $(p<0.05)$.

Conclusions: Surgery is an efficient option in treating GCSM, due to its efficacy in pain alleviation, function restoration, and stability reconstruction. Low levels of CEA, LDH, ALP, and high Tokuhashi score $(>6)$ are all favorable factors for better OS, whereas low levels of LDH, ALP, and application of bone cement are related with longer PFS.
\end{abstract}

Keywords: gastric cancer, spine metastasis, surgery

\section{Introduction}

Gastric cancer (GC) is the 5th most common cancer and the 2nd leading cause of cancer-related death worldwide. ${ }^{1}$ Hundahl et al have revealed a stage-stratified 3 -year survival rate as follows: ${ }^{2}$ stage IA $88.1 \%$, stage IB $77.6 \%$, stage IIA $71.2 \%$, stage IIB $58.8 \%$, stage IIIA $40.0 \%$, stage IIIB $28.0 \%$, stage IIIC $13.2 \%$, and stage IV $10.1 \%$. On account of advances in early detection and multidisciplinary treatment, life expectancies of GC patients have been prolonged. However, extended survival also leads to ascending incidence of tumor metastasis. Peritoneum, liver, and lung are the most common sites for metastasis, with bone metastasis being relatively scarce. The incidence of bone metastasis ranges from $0.9 \%$ to $12.4 \%$ in the literature, among which spine is the most commonly compromised. ${ }^{3-7}$

The presence of bone metastasis has been found to be an independent factor for dismal prognosis, with a median OS of 3-8 months after diagnosis. ${ }^{8-11}$ Furthermore, spinal metastasis may cause severe pain and neurological dysfunction, which exert a negative influence on the quality of patient's life. Thereafter, disease 
management in such cases remains challenging. Attributed to advances of surgical techniques and implant instruments, surgical intervention has become a promising option to achieve immediate pain alleviation, decompression, and stabilization.

The outcomes of surgical intervention for spinal metastasis have been thoroughly discussed. ${ }^{12}$ However, there is a paucity of published information on patients with gastric cancer spinal metastasis (GCSM) as a separate group. Park et al and Turkoz et al have analyzed the OS and prognostic factors for GC patients with bone metastasis, ${ }^{3,6}$ but none of the patients underwent surgical interventions. Therefore, in our research, we retrospectively reviewed GCSM patients surgically treated in our institution to present the therapeutic effect and long-term outcome for these patients.

\section{Materials and methods}

\section{Patients and follow-up strategy}

The research was approved by the hospital ethics committee, and written informed consent was obtained from all patients. In this study, patients with GCSM who received surgical treatments in our center between June 2011 and June 2017 were retrospectively reviewed. After excluding 2 patients who received surgeries merely for pathological biopsies, the data of a total of 17 patients were retrieved. Demographic information, serology tests, radiographic images, surgery records, and pathological results were all reviewed. All patients were assigned an Eastern Cooperative Oncology Group performance score (ECOG-PS) grade on admission, ${ }^{13}$ while Frankel score and visual analog scale (VAS) score were evaluated both preoperatively and postoperatively. All patients were followed every 3 months for the first 6 months after surgery, every 6 months for the following 1.5 years, and once a year afterward.

All patients enrolled have received surgical treatments after comprehensive assessments. Surgery indications include: 1) confirmed spinal metastasis with intolerable pain or neurological deficits and 2) conservative therapies could not achieve satisfying disease control. Besides, all patients who underwent surgeries were verified to have the ability to tolerate the proposed intervention based on their general conditions. Individualized surgical strategy was adopted for each patient based on the location and extent of tumors. After surgery, adjuvant therapies were delivered to patients according to their individual status.

\section{Statistical analysis}

Overall survival (OS) was defined as the time period from the date of surgery till the patients' death or the end of June 2018. Progression-free survival (PFS) was considered as the time period between the date of surgery and the date of local recurrence based on radiological assessments. Kaplan-Meier method was adopted to estimate survival rates, with log-rank test applied for univariate analysis. $P$ values less than 0.05 were considered statistically significant. All statistical calculations were performed by SPSS Statistics, version 21.0 (IBM Corp., New York, USA).

\section{Results}

\section{Patient description}

The median age of patients enrolled in our study is 56 (range: $30-72$ ), with a male/female ratio of 9/8. Skeletonrelated events (SREs) were observed in all patients, with pathological fracture observed in 5 patients, nocturnal pain in 16 patients, and neurologic deficits in 13 patients. Preoperative duration of symptoms ranged from 1 week to 6 months, with a median duration of 2 months. Pathology results revealed low differentiated signet-ring cell carcinoma in 8 patients and high-to-moderate differentiated adenocarcinoma in the rest 9 patients. Among all 17 patients, synchronous metastasis (development of spinal metastasis simultaneously with the diagnosis of GC) was identified in 9 patients, with the other 8 being metachronous (spinal metastasis occurs after the diagnosis of GC). All 8 patients have received chemotherapy before admission by our department: 4 patients were treated with FOLFOX regimen, 2 patients were treated with capecitabine and oxaliplatin, and 2 patients were treated with fluorouracil and oxaliplatin. Besides, 2 patients have received radiotherapy for spinal lesion and were later admitted by our department due to their progressive neural deficits. Locations of responsible metastatic lesions included 3 in the cervical spine, 9 in the thoracic spine, and 5 in the lumbar spine. Among all patients, 11 of them have metastasis of multilevel segments, either continuous or separated. In addition, extraspinal bone metastasis and visceral metastasis were noticed in 7 patients respectively.

\section{Treatment and prognosis}

All patients received total piecemeal resection to remove the bone lesions maximally. For 3 patients with cervical lesions, an anterior approach was adopted for resection and internal instrumentation, using titanium mesh filled 
with either bone cement or allograft bone. For patients with thoracic, lumbar and sacral metastasis, one-stage posterior approach was applied to achieve decompression, tumor removal, and internal fixation. Surgical field irrigation with oxaliplatin was performed for all patients. The mean surgery time was $244.4 \pm 84.0$ mins, while the mean blood loss during the operation was $1170.6 \pm 532.4 \mathrm{~mL}$. After surgery, 8 patients received bisphosphonate (zoledronic acid) to inhibit osteolysis, all patients were evaluated by the oncologist to receive or resume chemotherapy and 4 patients received adjuvant radiotherapy.

The median follow-up time was 11.6 months (range: $2-$ 21 months). At the end of the last follow-up, 14 patients were deceased, among whom, 12 died from metastasisrelated organ failure, 1 from gastric hemorrhage and another 1 from anesthesia-related enteroplegia. The median OS of all patients is 11.9 months (95\% CI: 8.4-15.4 months), while the median PFS is 11.3 months $(95 \% \mathrm{CI}$ : 7.8-14.9 months) (Figure 1). All patients had significant pain relief, with mean VAS score descending from $6.6 \pm 1.6$ to $3.4 \pm 1.2(p<0.001) 3$ months after surgery. Meanwhile, patients also showed improved neurological functions, with 8 patients having improvements of at least one level of Frankel classification (Figure 2). Additionally, patients enrolled also exhibited an improved general status, with mean ECOG-PS score descending from $2.44 \pm 1.21$ to 1.75 \pm 1.39 ( $p<0.05) 3$ months after surgery, which may save the patients from bedridden complications and enable them for further treatments. During the follow-up, 3 patients developed asymptomatic local recurrence, while 1 patient developed spinal metastasis of different segment 7 months after surgery. Surgical complications occurred in 2 patients, including one case of wound infection and another case of deep-vein thrombosis, and complete recovery was achieved after proper administration of antibiotic and anticoagulative therapy, respectively. Figure 3 illustrates the preoperative and postoperative radiologic records of a representative patient who was diagnosed to have cervical metastasis 5 years after diagnosis of GC. The patient received total piecemeal resection of the spinal lesion and postoperative adjuvant chemotherapy and experienced an OS of 19 months with a satisfactory quality of life.

\section{Univariate analysis of clinical factors}

The results of the univariate prognostic analysis are demonstrated in Table 1. Patients with a low level of LDH $(<300 \mathrm{U} / \mathrm{L})$ and ALP $(<200 \mathrm{U} / \mathrm{L})$ tend to have better OS and PFS $(p<0.05)$. Besides, a low level of CEA $(<6$ $\mu \mathrm{g} / \mathrm{L}$ ) is observed to be an indicator for better OS $(p=0.020)$. In the meanwhile, Tokuhashi scoring system also shows prognostic value in GCSM patients, with a score over 6 being related to a longer OS $(p=0.027)$. For local control, application of bone cement has shown with protective value, producing a median PFS of 17.7 months (95\% CI 11.6-23.8 months, $p=0.017$ ). Prognostic factors affecting OS and PFS are presented in Figure 4.

\section{Discussion}

Although bone metastasis is not common in GC patients, its presence may indicate poor prognosis. ${ }^{11}$ Park et al

A

B
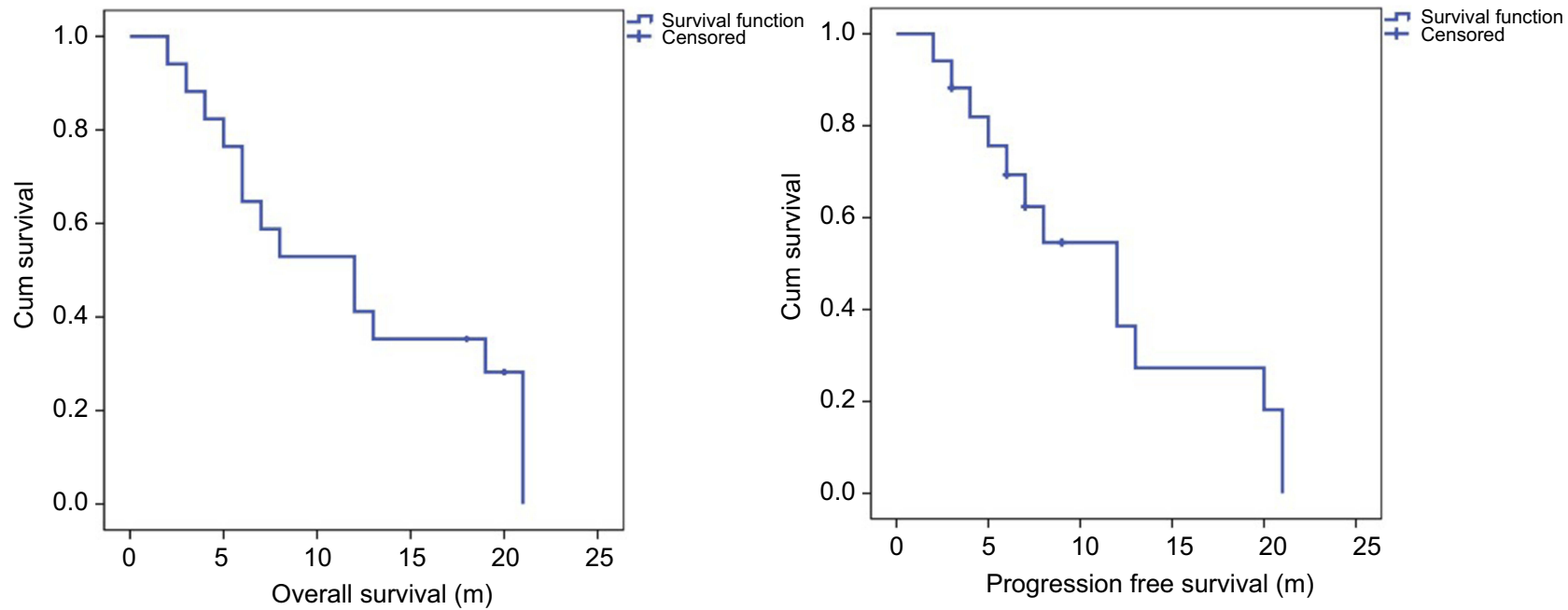

Figure I Overall survival and progression-free survival of all patients enrolled in our study. 


\begin{tabular}{c|c|c|c|c|c|c|}
\multicolumn{7}{c|}{ Postoperative frankel grade } \\
\cline { 2 - 7 } & A & A & B & C & D & E \\
\cline { 2 - 8 } & 0 & 1 & 1 & 0 & 0 \\
$\begin{array}{c}\text { Preoperative } \\
\text { frankel grade }\end{array}$ & B & 0 & 1 & 1 & 0 & 0 \\
\cline { 2 - 7 } & C & 0 & 0 & 1 & 3 & 1 \\
& D & 0 & 0 & 0 & 2 & 1 \\
\hline
\end{tabular}$\quad \square$ Improved

Figure 2 Preoperative and postoperative Frankel grade score.

analyzed 8633 Korean GC patients, among which 204 (2.4\%) patients were identified to have bone metastasis. ${ }^{3}$ All 204 patients received either chemotherapy or supportive care, and ultimately achieved a median OS of 103 days. In another research, Turkoz et al reviewed 4617 GC patients, with 176 (3.8\%) of them burdened with bone metastasis. ${ }^{6}$ After treatment of either chemotherapy or radiotherapy, a median OS of 5.4 months was obtained. Notably, none of the patients enrolled in the above studies underwent surgical treatment for spinal metastasis.

Meta-analysis and prospective trial have demonstrated the benefits of surgery in treating spinal metastasis, including better ambulation, pain alleviation as well as longer OS. ${ }^{14,15}$ However, there has been a long-standing discussion about whether surgery remains beneficial for such aggressive carcinoma as GC. To the best of our knowledge, no study has evaluated the efficacy of surgery with other treating modalities in GCSM. Chong et al analyzed 105 patients who underwent single-stage posterior decompression and stabilization for thoracic spinal metastasis. ${ }^{16}$ And the median survival of patients with rapid growth tumor type (including 45 lung cancer, 26 hepatobiliary cancer, $4 \mathrm{GC}$, and 3 esophageal cancer) was 6 months. In our study, all patients received total piecemeal spondylectomy and internal instrumentation, and the median OS
A

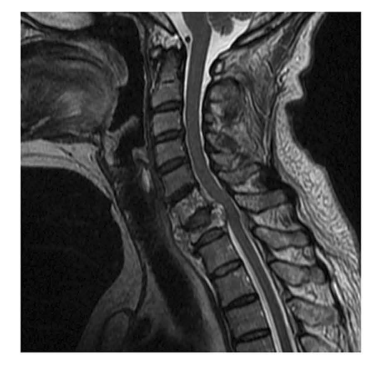

C

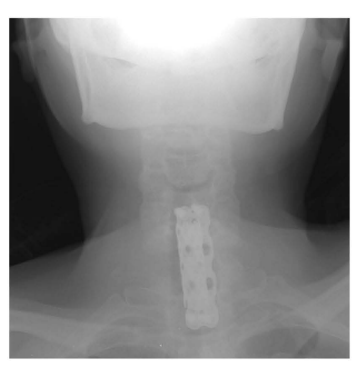

E

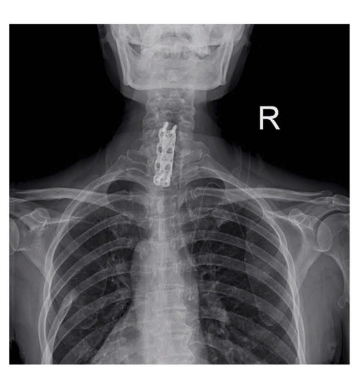

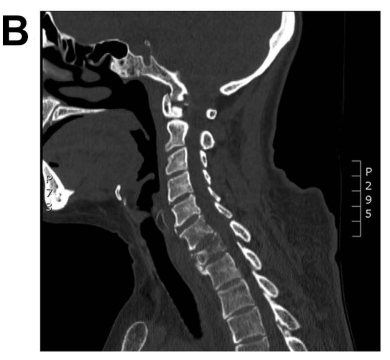

D

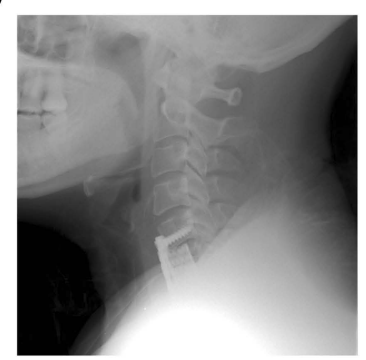

$\mathbf{F}$

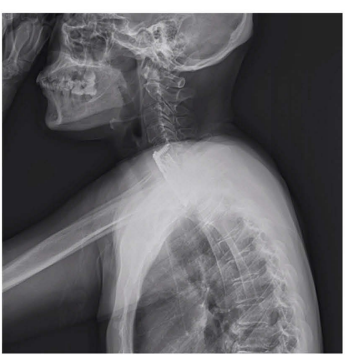

Figure 3 Radiographic images of a representative 55-year-old female patient (Case \#I5).

Notes: Preoperative MRI (A) and CT (B) showing destruction of C6-7 vertebrae with instability and spinal cord compression. (C-D) Postoperative radiograph after total piecemeal spondylectomy and internal fixation using titanium mesh and plate osteosynthesis system. (E-F) X-ray image at 23 months after surgery showing no local relapse and stable instrumentation.

Abbreviations: MRI, magnetic resonance imaging; CT, computed tomography. 
Table I Univariate analysis of prognostic factors affecting OS

\begin{tabular}{|c|c|c|c|}
\hline Factor & No. & $P_{(\text {os) }}$ & $P_{(\text {PFS })}$ \\
\hline Age $\leq 50$ years $/>50$ years & $5 / 12$ & 0.313 & 0.081 \\
\hline Gender: male/female & $9 / 8$ & 0.834 & 0.691 \\
\hline $\begin{array}{l}\text { Duration of symptom: } \leq 2 \text { months/>2 } \\
\text { months }\end{array}$ & $8 / 9$ & 0.269 & 0.364 \\
\hline Chronology: syn/meta & $9 / 8$ & 0.520 & 0.488 \\
\hline $\begin{array}{l}\text { Pathology: moderate-high/low } \\
\text { differentiation }\end{array}$ & $9 / 8$ & 0.410 & 0.098 \\
\hline Spinal metastasis: local/multiple & $6 / 11$ & 0.134 & 0.248 \\
\hline Spinal metastasis: cervical/thoracic/lumbar & $3 / 9 / 5$ & 0.682 & 0.697 \\
\hline Extraspinal bone metastasis: no/yes & $9 / 8$ & 0.722 & 0.993 \\
\hline Visceral metastasis: no/yes & $9 / 8$ & 0.323 & 0.950 \\
\hline Preoperative ECOG-PS: $0-2 / 3-5$ & $9 / 8$ & 0.295 & 0.399 \\
\hline $\mathrm{LDH}:<300 / \geq 300 \mathrm{U} / \mathrm{L}$ & $6 / 11$ & $0.012^{*}$ & $0.016^{*}$ \\
\hline ALP: $<200 / \geq 200 \mathrm{U} / \mathrm{L}$ & $10 / 7$ & $0.007^{*}$ & $0.034^{*}$ \\
\hline CEA: $<6 / \geq 6 \mu g / L$ & $9 / 8$ & $0.020 *$ & 0.083 \\
\hline CAI9-9: $<50 / \geq 50 \mathrm{U} / \mathrm{mL}$ & $8 / 9$ & 0.075 & 0.280 \\
\hline Tokuhashi score: $\leq 6 />6$ & $10 / 7$ & $0.027^{*}$ & 0.136 \\
\hline Tomita score: $<8 / \geq 8$ & $9 / 8$ & 0.279 & 0.555 \\
\hline Surgery time: $<4 / \geq 4$ hrs & $7 / 10$ & 0.770 & 0.421 \\
\hline Blood loss: $<1000 / \geq 1000 \mathrm{~mL}$ & $6 / 11$ & 0.885 & 0.974 \\
\hline Gastrectomy: no/yes & $12 / 5$ & 0.216 & 0.355 \\
\hline Bone cement: no/yes & $7 / 10$ & 0.083 & $0.017^{*}$ \\
\hline Chemotherapy: no/yes & $8 / 9$ & 0.121 & 0.156 \\
\hline Radiotherapy: no/yes & $13 / 4$ & 0.205 & 0.474 \\
\hline Bisphosphonate: no/yes & $9 / 8$ & 0.637 & 0.569 \\
\hline
\end{tabular}

Note: $* P$-values $<0.05$ were considered statistically significant.

is 11.9 months (95\% CI: $8.4-15.4$ months). Compared to the OS of patients treated merely with conservative therapeutic modalities as shown in the literature, a prolongation of life expectancy was detected in our cohort. This advantage could be ascribed to the following reasons: 1) spinal surgery can efficiently improve the functional status of patients, thus affording patients more chances to receive postoperative adjuvant therapies; 2) surgery can achieve better ambulation, which can prevent patients from bedridden complications such as pulmonary infection and deep-venous thrombosis, etc.

For all 17 patients enrolled in our study, total piecemeal spondylectomy and further internal instrumentation are applied. Although it has been widely accepted that total en bloc spondylectomy (TES) is an ideal option to control local recurrence of spinal malignancy, ${ }^{17-19}$ the indication to perform TES should be individually weighed on the risk-tobenefit ratio in the situation of spine metastasis. First, TES is not always feasible due to the constraints of anatomic structure. For instance, lesions of the cervical and lower lumbar segment and paravertebral involvement may pose barriers to fulfill a standard en bloc resection. Contrarily, solitary intracompartmental metastatic lesions are potential candidates to receive this locally curative treatment. ${ }^{20}$ Other factors that needed to be evaluated are the disease progression and the general condition of patients. Given the possible excessive morbidity, blood loss, and operative time of TES resulted from its great technical demands, it was concluded that TES should be reserved for selected patients with controllable primary tumors, localized metastatic lesions, and expected long-term survival to ensure the improvement in prognosis. ${ }^{21}$ In the current retrospective cohort, we choose total piecemeal spondylectomy for all the patients instead of TES after a thorough assessment of the aspects mentioned earlier. We also suggested the irrigation of surgical fields with oxaliplatin to eliminate any possible tumor residues. Moreover, the application of bone cement is observed to be relevant to better local control, with $p=0.016$ for PFS. This possible antitumor effect of bone cement may be ascribed to its cytotoxic and thermal effects. ${ }^{22}$ Taken together, our follow-up data showed a generally satisfied incidence of surgical complications and long-term outcome. Nevertheless, it is worth comparing the therapeutic efficacy between TES, total piecemeal spondylectomy, and other less radical resection methods when handling high-grade metastatic malignancies such as GCSM.

In our study, several favorable prognostic factors for postoperative outcome have been identified, including $\mathrm{CEA}<6 \quad \mu \mathrm{g} / \mathrm{L} \quad(p=0.020), \quad \mathrm{LDH}<300 \quad \mathrm{U} / \mathrm{L} \quad(p=0.012)$, ALP $<200 \quad \mathrm{U} / \mathrm{L} \quad(p=0.007)$, and Tokuhashi score $>6$ $(p=0.027)$. Tumor antigens have been extensively applied for tumor detection and can, to some extent, reflect tumor burden and disease progression. In the case of GC, the most commonly used serum markers include CEA, CA199, and CA72-4, which have been proven prognostic for disease progression in multiple clinical trials and metaanalysis. ${ }^{23-27}$ The present results also supported a similar prognostic potency of these biomarkers for GCSM. Besides, LDH and ALP, serving as crucial enzymes in oncogenesis and bone metastasis, have also been claimed of prognostic value in GC patients. ${ }^{28-30}$ Our findings confirmed the previous reports regarding the role of LDH and ALP in metastases, suggesting that patients with ALP $<200$ $\mathrm{U} / \mathrm{L}$ and $\mathrm{LDH}<300 \mathrm{U} / \mathrm{L}$ tend to have a longer OS and PFS $(p<0.05)$. Presence of visceral metastasis is typically considered as an indicator for dismal prognosis in GCSM patients. ${ }^{8,9}$ In our series, the OS for patients with or without visceral metastasis is $10.5 \pm 2.5$ months and $13.1 \pm 2.7$ months, respectively, with no statistical significance being observed $(p=0.323)$. Nonetheless, Tokuhashi score, which 

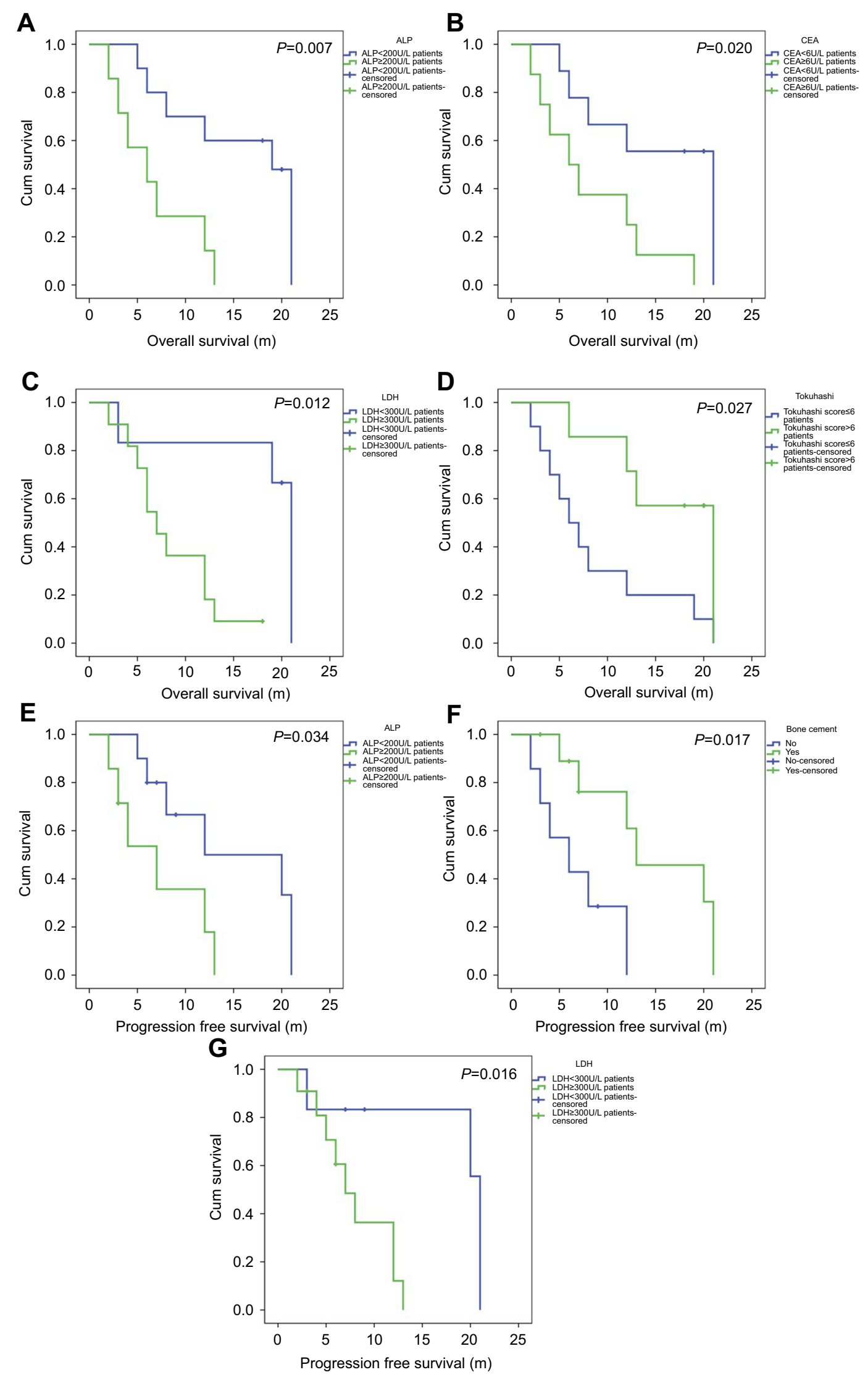

Figure 4 Univariate analysis of prognostic factors affecting OS and PFS significantly. 
integrated 6 parameters of different angles, including visceral metastasis, ${ }^{31}$ has shown prognostic value in predicting survival $(p=0.027)$. Generally, our results suggest that GCSM patients with the above characteristics may possess a better outcome after surgical intervention.

Apart from surgery, we also assessed the potential advantage of adjuvant therapies in treating patients with GCSM. Radiotherapy is typically considered beneficial in treating spinal metastasis, especially in pain alleviation and local control of spinal lesions. ${ }^{32-34}$ However, the ultimate amelioration rate is fundamentally dependent on the tumor's inherent radiosensitivity. In our study, patients who received postoperative radiotherapy do not exhibit a longer PFS $(11.9 \pm 2.0$ vs $6.7 \pm 1.5, p=0.474)$, probably due to the small scale of patients enrolled in our study and the insensitive nature of $\mathrm{GCSM}^{35}$ As for chemotherapy, Nakamura et $\mathrm{al}^{9}$ reviewed $25 \mathrm{GC}$ patients suffering from bone metastasis with concomitant extraosseous metastasis and observed a longer OS in those receiving systemic chemotherapy (125 days vs 21 days, $p=0.001$ ). However, such benefits are not observed in our research $(14.5 \pm 2.8$ months vs $9.4 \pm 2.0$ months, $p=0.121$ ). Overall, the outcome of systemic chemotherapy for GCSM patients remains controversial due to its low response rate and severe side effects. ${ }^{11}$ Although the optimal adjuvant therapeutic regimens for GCSM are yet to be judged, individualized treatment tailored for patients with various conditions might be a reasonable choice.

Patients with GCSM tend to suffer from SREs during the late course of their disease. Bisphosphonate, known for inhibition of osteoclast-mediated bone resorption and potential antitumor effect, has been used to prevent SREs over the past decades in the systemic treatment of bone metastasis. ${ }^{36,37}$ Silvestris et al investigated 186 GC patients with bone metastasis and reported an extension of time till first SRE among the 81 patients treated with bisphosphonate. ${ }^{5}$ Meanwhile, the 81 patients also exhibit a longer OS (8 months vs 5 months, $p=0.001$ ), with no significant adverse events being observed during the treatment. In our study, although bisphosphonate does not show significant role in prolonging OS $(p=0.637)$ or PFS ( $p=0.569)$, among the 9 patients who did not receive bisphosphonate, 4 of them developed local relapse during the follow-up, whereas no local recurrence occurred in the patients treated with zoledronic acid.

\section{Conclusion}

The current data supported that surgery is a feasible therapeutic option for selected GCSM patients. Surgery has been proven to be effective in pain alleviation and function preservation. According to univariate analysis, low level of CEA, LDH, ALP, and high Tokuhashi score may be considered as beneficial prognostic factors for GCSM patients. Nonetheless, there are yet some limitations to our study. Due to the low incidence of GCSM, there are only 17 patients enrolled in our study; thus, the power of conclusions may be diminished. In addition, there is only 1 patient receiving targeted medications in our study, which hampered the analysis of the adjuvant target therapy. Thereafter, a study examining a large cohort is required to be carried out to validate our findings and attain a more comprehensive evaluation of the GCSM therapeutic strategy.

\section{Ethics}

This study was reviewed and approved by the Ethics Committee of Changzheng Hospital of Naval Medical University. Written informed consent was obtained by the patients, in compliance with the Declaration of Helsinki.

\section{Acknowledgments}

We are grateful to the patients for their cooperation and to all of the clinicians and support staff who were involved in caring for the patients involved in this study in the Department of Orthopedic Oncology and Spinal Tumor Center, Shanghai Changzheng Hospital. The research was generously supported by the Shanghai Science and Technology Committee (Grant No. 17411950300), the Shanghai Sailing Program (18YF-1423000), and the Foundation for Young Scholars of Naval Medical University (2017QN16, 2917QN17).

\section{Disclosure}

The authors report no conflicts of interest in this work.

\section{References}

1. Torre LA, Bray F, Siegel RL, Ferlay J, Lortet-Tieulent J, Jemal A. Global cancer statistics, 2012. CA Cancer J Clin. 2015;65(2):87-108. doi:10.3322/caac. 21262

2. In H, Solsky I, Palis B, Langdon-Embry M, Ajani J, Sano T. Validation of the 8th edition of the AJCC TNM staging system for gastric cancer using the national cancer database. Ann Surg Oncol. 2017;24 (12):3683-3691. doi:10.1245/s10434-017-6078-x

3. Park HS, Rha SY, Kim HS, et al. A prognostic model to predict clinical outcome in gastric cancer patients with bone metastasis. Oncology. 2011;80(1-2):142-150. doi:10.1159/000328507

4. Ahn JB, Ha TK, Kwon SJ. Bone metastasis in gastric cancer patients. $J$ Gastric Cancer. 2011;11(1):38-45. doi:10.5230/jgc.2011.11.1.38 
5. Silvestris N, Pantano F, Ibrahim T, et al. Natural history of malignant bone disease in gastric cancer: final results of a multicenter bone metastasis survey. Plos One. 2013;8(10):e74402. doi:10.1371/journal. pone.0074402

6. Turkoz FP, Solak M, Kilickap S, et al. Bone metastasis from gastric cancer: the incidence, clinicopathological features, and influence on survival. J Gastric Cancer. 2014;14(3):164-172. doi:10.5230/ jgc.2014.14.3.164

7. Qiu MZ, Shi SM, Chen ZH, et al. Frequency and clinicopathological features of metastasis to liver, lung, bone, and brain from gastric cancer: a SEER-based study. Cancer Med. 2018. doi:10.1002/ cam4.1661

8. Mikami J, Kimura Y, Makari Y, et al. Clinical outcomes and prognostic factors for gastric cancer patients with bone metastasis. World J Surg Oncol. 2017;15(1):8. doi:10.1186/s12957-016-1091-2

9. Nakamura K, Tomioku $M$, Nabeshima $K$, Yasuda $S$. Clinicopathologic features and clinical outcomes of gastric cancer patients with bone metastasis. Tokai J Exp Clin Med. 2014;39 (4):193-198.

10. Choi YJ, Kim DH, Han HS, et al. Long-term survival after gastrectomy and metastasectomy for gastric cancer with synchronous bone metastasis. World J Gastroenterol. 2018;24(1):150-156. doi:10.3748/ wjg.v24.i1.150

11. Lee J, Lim T, Uhm JE, et al. Prognostic model to predict survival following first-line chemotherapy in patients with metastatic gastric adenocarcinoma. Ann Oncol. 2007;18(5):886-891. doi:10.1093/ annonc/mdl501

12. Choi D, Crockard A, Bunger C, et al. Review of metastatic spine tumour classification and indications for surgery: the consensus statement of the Global Spine Tumour Study Group. Eur Spine J. 2010;19 (2):215-222. doi:10.1007/s00586-009-1252-x

13. Oken MM, Creech RH, Tormey DC, et al. Toxicity and response criteria of the Eastern Cooperative Oncology Group. Am J Clin Oncol. 1982;5(6):649-655. doi:10.1097/00000421-198212000-00014

14. Klimo PJ, Thompson CJ, Kestle JR, Schmidt MH. A meta-analysis of surgery versus conventional radiotherapy for the treatment of metastatic spinal epidural disease. Neuro Oncol. 2005;7(1):64-76. doi:10.1215/S1152851704000262

15. Patchell RA, Tibbs PA, Regine WF, et al. Direct decompressive surgical resection in the treatment of spinal cord compression caused by metastatic cancer: a randomised trial. Lancet. 2005;366 (9486):643-648. doi:10.1016/S0140-6736(05)66954-1

16. Chong S, Shin SH, Yoo H, et al. Single-stage posterior decompression and stabilization for metastasis of the thoracic spine: prognostic factors for functional outcome and patients' survival. Spine J. 2012;12(12):1083-1092. doi:10.1016/j.spinee.2012.10.015

17. Demura S, Kawahara N, Murakami H, et al. Total en bloc spondylectomy for spinal metastases in thyroid carcinoma. J Neurosurg Spine. 2011;14(2):172-176. doi:10.3171/2010.9.SPINE09878

18. Yang X, Wu Z, Xiao J, et al. Chondrosarcomas of the cervical and cervicothoracic spine: surgical management and long-term clinical outcome. J Spinal Disord Tech. 2012;25(1):1-9. doi:10.1097/ BSD.0b013e31820bb085

19. Jia Q, Yin H, Yang J, et al. Treatment and outcome of metastatic paraganglioma of the spine. Eur Spine J. 2018;27(4):859-867. doi:10.1007/s00586-017-5140-5

20. Domenicucci M, Nigro L, Delfini R. Total en-bloc spondylectomy through a posterior approach: technique and surgical outcome in thoracic metastases. Acta Neurochir (Wien). 2018;160(7):13731376. doi:10.1007/s00701-018-3572-2
21. Park JH, Hyun SJ, Kim KJ, Jahng TA. Total en bloc thoracic and lumbar spondylectomy for non-small cell lung cancer with favorable prognostic indicators: is it merely indicated for solitary spinal metastasis? J Korean Neurosurg Soc. 2014;56(5):431-435. doi:10.3340/ jkns.2014.56.5.431

22. Roedel B, Clarencon F, Touraine S, et al. Has the percutaneous vertebroplasty a role to prevent progression or local recurrence in spinal metastases of breast cancer? J Neuroradiol. 2015;42(4):222228. doi:10.1016/j.neurad.2014.02.004

23. Tocchi A, Costa G, Lepre L, et al. The role of serum and gastric juice levels of carcinoembryonic antigen, CA19.9 and CA72.4 in patients with gastric cancer. J Cancer Res Clin Oncol. 1998;124(8):450-455.

24. Tachibana M, Takemoto Y, Nakashima Y, et al. Serum carcinoembryonic antigen as a prognostic factor in resectable gastric cancer. $J$ Am Coll Surg. 1998;187(1):64-68.

25. Ychou M, Duffour J, Kramar A, Gourgou S, Grenier J. Clinical significance and prognostic value of CA72-4 compared with CEA and CA19-9 in patients with gastric cancer. Dis Markers. 2000;16(34):105-110. doi:10.1155/2000/595492

26. Ishigami $\mathrm{S}$, Natsugoe $\mathrm{S}$, Hokita $\mathrm{S}$, et al. Clinical importance of preoperative carcinoembryonic antigen and carbohydrate antigen 19-9 levels in gastric cancer. J Clin Gastroenterol. 2001;32(1):41-44.

27. Deng K, Yang L, Hu B, Wu H, Zhu H, Tang C. The prognostic significance of pretreatment serum CEA levels in gastric cancer: a meta-analysis including 14651 patients. Plos One. 2015;10(4):e124151.

28. Sun X, Sun Z, Zhu Z, et al. Clinicopathological significance and prognostic value of lactate dehydrogenase A expression in gastric cancer patients. Plos One. 2014;9(3):e91068. doi:10.1371/journal. pone.0091068

29. Li G, Gao J, Tao YL, et al. Increased pretreatment levels of serum LDH and ALP as poor prognostic factors for nasopharyngeal carcinoma. Chin J Cancer. 2012;31(4):197-206. doi:10.5732/cjc.011.10283

30. Han J, Yong B, Luo C, Tan P, Peng T, Shen J. High serum alkaline phosphatase cooperating with MMP-9 predicts metastasis and poor prognosis in patients with primary osteosarcoma in Southern China. World J Surg Oncol. 2012;10:37. doi:10.1186/1477-7819-10-198

31. Tokuhashi Y, Matsuzaki H, Oda H, Oshima M, Ryu J. A revised scoring system for preoperative evaluation of metastatic spine tumor prognosis. Spine (Phila Pa 1976). 2005;30(19):2186-2191.

32. Yoshikawa K, Kitaoka H. Bone metastasis of gastric cancer. Jpn J Surg. 1983;13(3):173-176.

33. Murai N, Koga K, Nagamachi S, et al. Radiotherapy in bone metastases-with special reference to its effect on relieving pain. Gan No Rinsho. 1989;35(10):1149-1152.

34. Rades D, Lange M, Veninga $\mathrm{T}$, et al. Final results of a prospective study comparing the local control of short-course and long-course radiotherapy for metastatic spinal cord compression. Int J Radiat Oncol Biol Phys. 2011;79(2):524-530. doi:10.1016/j. ijrobp.2009.10.073

35. Laufer I, Rubin DG, Lis E, et al. The NOMS framework: approach to the treatment of spinal metastatic tumors. Oncologist. 2013;18 (6):744-751. doi:10.1634/theoncologist.2012-0293

36. Wang HJ, Liu Y, Fan LQ, et al. A new bisphosphonate derivative, CP, induces gastric cancer cell apoptosis via activation of the ERK1/2 signaling pathway. Acta Pharmacol Sin. 2013;34(12):1535-1544. doi:10.1038/aps.2013.103

37. Yang H, Qiu L, Zhang L, et al. Platinum-zoledronate complex blocks gastric cancer cell proliferation by inducing cell cycle arrest and apoptosis. Tumour Biol. 2016;37(8):10981-10992. doi:10.1007/ s13277-016-4977-2 


\section{Publish your work in this journal}

Cancer Management and Research is an international, peer-reviewed open access journal focusing on cancer research and the optimal use of preventative and integrated treatment interventions to achieve improved outcomes, enhanced survival and quality of life for the cancer patient.
The manuscript management system is completely online and includes a very quick and fair peer-review system, which is all easy to use. Visit http://www.dovepress.com/testimonials.php to read real quotes from published authors.

Submit your manuscript here: https://www.dovepress.com/cancer-management-and-research-journal 